\title{
Influence of SULTIAI*2 Polymorphism on Plasma Efavirenz Concentration in Thai HIV-I Patients
}

\author{
Monpat Chamnanphon $\mathbb{D}^{1,2, *}$ \\ Rattanaporn Sukprasong ${ }^{3,4, *}$ \\ Andrea Gaedigk (D) ${ }^{5,6}$ \\ Weerawat Manosuthi ${ }^{7}$ \\ Pajaree Chariyavilaskul (D) ${ }^{2}$ \\ Supeecha Wittayalertpanya ${ }^{2}$ \\ Napatrupron Koomdee 3,4 \\ Thawinee Jantararoungtong ${ }^{3,4}$ \\ Apichaya Puangpetch ${ }^{3,4}$ \\ Chonlaphat Sukasem ${ }^{3,4}$ \\ 'Department of Pathology, Faculty of \\ Medicine, Srinakharinwirot University, \\ Nakornnayok, Thailand; ${ }^{2}$ Clinical \\ Pharmacokinetics and Pharmacogenomics \\ Research Unit, Department of \\ Pharmacology, Faculty of Medicine, \\ Chulalongkorn University, Bangkok, \\ Thailand; ${ }^{3}$ Division of Pharmacogenomics \\ and Personalized Medicine, Department \\ of Pathology, Faculty of Medicine \\ Ramathibodi Hospital, Mahidol \\ University, Bangkok, Thailand; \\ ${ }^{4}$ Laboratory for Pharmacogenomics, \\ Somdech Phra Debaratana Medical \\ Center (SDMC), Ramathibodi Hospital, \\ Bangkok, Thailand; ${ }^{5}$ Division of Clinical \\ Pharmacology, Toxicology \& Therapeutic \\ Innovation, Children's Mercy Kansas City, \\ Kansas City, MO, USA; ${ }^{6}$ School of \\ Medicine, University of Missouri-Kansas \\ City, Kansas City, MO, USA: \\ ${ }^{7}$ Bamrasnaradura Infectious Diseases \\ Institute, Ministry of Public Health, \\ Nonthaburi, Thailand
}

*These authors contributed equally to this work

Correspondence: Chonlaphat Sukasem Division of Pharmacogenetics and Personalized Medicine, Department of Pathology, Faculty of Medicine,

Ramathibodi Hospital, Mahidol University, Bangkok, 10400, Thailand

Tel +66-2-200-433I

Fax +66-2-200-4332

Email chonlaphat.suk@mahidol.ac.th
Purpose: Plasma efavirenz (EFV) concentrations within therapeutic levels are essential to successfully treat patients suffering from human immunodeficiency virus (HIV) type 1 . In addition to the drug-metabolizing enzyme CYP2B6, other phase II drug-metabolizing enzymes and transporters may have an important role in the pharmacokinetics of EFV. Thus, the influence of phase II drug-metabolizing enzymes and drug transporters on plasma EFV levels was investigated in Thai HIV patients receiving EFV.

Patients and Methods: Genotyping was performed by TaqMan ${ }^{\circledR}$ real-time PCR in 149 HIV-infected Thai adults, and plasma efavirenz concentration was measured by a validated high-performance liquid chromatography in 12 hours after dosing steady-state plasma samples at week 12 and 24.

Results: Patients with three or more copies of SULT1A1 had significantly lower median plasma EFV concentrations than those carrying two copies at week $12(p=0.046)$ and SULT1A1*2 (c.638G $>$ A) carriers had significantly lower median plasma EFV concentrations compared to those not carrying the variant at week $24(p=0.048)$. However, no significant association was found after adjusting for $C Y P 2 B 6$ genotype.

Conclusion: Genetic variation in a combination of SULT1A1*2 and SULT1A1 copy number may contribute to variability in EFV metabolism and thereby may impact drug response. The influence of a combination between the SULT1A1 and CYP2B6 genotype on EFV pharmacokinetics should be further investigated in a larger study population.

Keywords: phase II drug-metabolizing enzymes, transporter genes, efavirenz, HIV-1, Thai

\section{Introduction}

Human immunodeficiency virus type 1 infection (from here on forward referred to "HIV") is a major global health problem including Thailand. Co-infections with other viruses including hepatitis $\mathrm{B}$ are also common. ${ }^{1}$ Efavirenz (EFV), a nonnucleoside reverse transcriptase inhibitor (NNRTI) is a mainstay component in highly active antiretroviral therapy (HAART). EFV is combined with Truvada, which consists of tenofovir and emtricitabine; this triple combination provides the principal HAART in a single, once a day tablet to effectively suppress HIV replication in the majority of patients. ${ }^{2}$ This drug combination has been approved by the US Food and Drug Administration (FDA) in July 2006 under the brand name Atripla and is listed as one of the most important medications needed in basic health systems in the Essential Medicines List issued by the World Health Organization. The preferred therapeutic range of EFV plasma concentrations is $1-4 \mathrm{mg} / \mathrm{L}$. Plasma concentrations below $1 \mathrm{mg} / \mathrm{L}$ have been associated with virological failure, ${ }^{3-8}$ effectively causing a patient to have "treatment failure on an EFV-based 
regimen". 6 In contrast, plasma concentrations $>4 \mathrm{mg} / \mathrm{L}$ have been associated with a higher risk of central nervous system (CNS) side effects, ${ }^{6,8}$ as well as CNS toxicity that often causes patients to discontinue therapy. $3,4,6,7,9-13$

CYP2B6 plays a major role in EFV metabolism. ${ }^{14}$ Several previous studies, including our cohort, have reported the influence of CYP2B6 enzyme on EFV pharmacokinetics. Increased plasma EFV concentrations were associated with $C Y P 2 B 6$ genotype in HIV patients. ${ }^{12,15,16}$ A few studies to date have investigated only CYP2B6 and plasma EFV concentrations, however, other enzymes such as CYP3A4/5, CYP1A2 and CYP2A6 in minor pathways $^{17,18}$ are still overlooked. Furthermore, EFV is directly conjugated with glucuronic acid via UDPglucuronosyltransferase (UGT) 2B7 into EFV$N$-glucuronide, and hydroxyefavirenz metabolites can be further glucuronidated by UGTs shunting them towards urinary excretion. ${ }^{19-21}$ In addition, an association between single nucleotide polymorphisms (SNPs) in the $A B C C 4$ c.3348A $>\mathrm{G}$ (rs1751034), c.912G $>\mathrm{T}(\mathrm{rs} 2274407)$ and EFV kinetics was reported. $^{22}$ This observation was consistent with an in vitro study describing an association of $A B C C 4$ c.559G $>\mathrm{T}$ (rs11568658) and c.1460A $>\mathrm{G}$ (rs11568668) and intracellular accumulation of azidothymidine (AZT). ${ }^{23}$ Finally, Belanger et al showed that UGT2B7 can directly conjugate EFV to EFV-N-glucuronide (EFV$\mathrm{G}),{ }^{21}$ and that a synonymous $U G T 2 B 7 \quad$ c. $735 \mathrm{~A}>\mathrm{G}$ (rs28365062) SNP that is part of some $U G T 2 B 7 * 1$ suballeles as well as the $U G T 2 B 7^{*} 4$ allele was associated with increased EFV concentrations ${ }^{24}$ contrasting other investigations. $^{25-27}$ SULT1A1 has also been reported to influence EFV metabolism. ${ }^{24}$ It has never been investigated, however, whether SULT1A1 and/or UGT2B17 gene copy number variation (CNV) impact EFV concentrations.

Because there is sparse or no data regarding the impact of phase II drug-metabolizing enzymes and drug transporters on EFV metabolism. We aimed to investigate the influence of sequence variations and copy number in these genes on plasma efavirenz concentrations in Thai HIV-infected adult patients.

\section{Materials and Methods \\ Patient Samples}

One hundred and forty-nine HIV-infected adult Thai patients receiving EFV were recruited from the outpatient unit of the Bamrasnaradura Infectious Diseases Institute, Ministry of Public Health and Nonthaburi, Thailand. All subjects provided written informed consent to participate in the study in accordance with the guidelines of the Declaration of Helsinki. This study was approved by the Ethics Committee of the Faculty of Medicine Ramathibodi Hospital, Mahidol University, Thailand. Enrolled subjects for the study were over 18 years, had no opportunistic infection and receiving efavirenz $600 \mathrm{mg}$, tenofovir $300 \mathrm{mg}$, lamivudine $300 \mathrm{mg}$ at bedtime. Mid-dose efavirenz plasma concentration was measured at 12 and 24 weeks following initiation of antiretroviral therapy. Patients receiving concomitant treatments that could potentially affect efavirenz pharmacokinetics were excluded. And the CYP2B6 genotype results from our previous studies, ${ }^{15,28,29}$ which consisted of $* 1 / * 1(52,34.9 \%), * 1 / * 2(11,7.4 \%), * 1 /$ $* 4(3,2.0 \%), * 1 / * 6(61,40.9 \%), * 2 / * 4(2,1.3 \%), * 2 / * 6(2$, $1.3 \%), * 4 / * 4(5,3.4 \%), * 5 / * 6(2,1.3 \%), * 6 / * 6(11,7.4 \%)$, were used for calculation in multiple regression analysis to control the impact of major CYP2B6 enzyme that may interfere the results in this study.

\section{Genotype of Phase II Drug Metabolizing Enzymes and Transporter Genes}

DNA was isolated from the stored EDTA cell pellets $\left(-20^{\circ} \mathrm{C}\right)$ using the QIAamp ${ }^{\circledR}$ DNA Blood Mini Kit (Qiagen, Hilden, Germany). Genomic DNA was quantified by a UV spectrophotometer ND-1000 at $260 \mathrm{~nm}$ (NanoDrop Technologies, Wilmington, DE).

Genotyping with TaqMan ${ }^{\circledR}$ assays was performed on a ViiA7 real-time PCR instrument (Applied Biosystems, Foster City, CA, USA) as previously described. ${ }^{28}$ Genotyping included eight SNPs ( $\mathrm{rs}$ and assay IDs are shown in brackets): $A B C A 1$ c. $4760 \mathrm{~A}>\mathrm{G}$ (rs2230808, C_2741104_1_), $A B C C 2 \quad$ g.68231A $>\mathrm{G} \quad$ (rs3740065, C_22271640_10), ABCC2 c. $24 \mathrm{C}>\mathrm{T} \quad$ (rs717620, C_2814642_10), $A B C C 4 \quad$ c.3348A $>\mathrm{G} \quad$ (rs1751034, C_1901918_30), SULT1A1 c.638G $>$ A (rs1042028 or rs9282861, AHOJH16), UGT2B7 c.-161C $>$ T (rs7668258, C_27827970_40, part of some $U G T 2 B 7^{*} 1$ haplotypes and *2), UGT2B7 c. $211 \mathrm{G}>\mathrm{T}$ (rs12233719, C_45181106_10, $U G T 2 B 7^{*} 3$ ) and $U G T 2 B 7$ c. $372 \mathrm{~A}>\mathrm{G}$ (rs28365063, C_30689135_20, found in some $U G T 2 B 7^{*} 1$ and *3).

\section{SULTIAI and UGT2BI7 Copy Number Variation Analysis}

$\mathrm{CNV}$ analysis was performed by quantitative multiplex PCR amplification (MPA) as previously described by Gaedigk et al. ${ }^{30}$ Briefly, PCR fragments were separated 
on an ABI 3730 instrument (Applied Biosystems, Foster City, CA, USA) and analyzed with GeneMapper ${ }^{\circledR}$. UGT2B17 and SULT1A1 copy number was determined by normalizing against UGT2B15 and SULT1A2, respectively.

\section{Measurement of Efavirenz Plasma Concentrations}

Fasting plasma EFV concentrations 12 hours after dosing were measured at 12 and 24 weeks following antiretroviral therapy initiation. A validated isocratic reversed-phase highperformance liquid chromatography (HPLC) method with ultraviolet detection at $245 \mathrm{~nm}$ was used to measure plasma EFV concentrations as previously described. ${ }^{29}$ Plasma samples ( $300 \mu \mathrm{L}$ pretreated with acetonitrile) were injected into an Agilent 1100 HPLC instrument equipped with an Omnispher C18 (150 x $4.6 \mathrm{~mm} \mathrm{ID/particle} \mathrm{size} 5 \mu \mathrm{m})$ analytical column (Varian, CA, and USA), and a ChromGuard RP guard column. The mobile phase consisted of $10 \mathrm{mM} \mathrm{KH}_{2}$ $\mathrm{PO}_{4} \mathrm{pH}$ 3.1: acetonitrile (50: 50, v/v). ChromQuest Software version 4.1 was used for processing the sample peak heights. The average accuracy was $102-105 \%$ and the coefficient of variation was $<5 \%$.

\section{Statistical Analysis}

Genotype distributions were tested for Hardy-Weinberg equilibrium using exact tests under a call rate of $95 \%$ exemption. Data were summarized using medians and interquartile ranges (IQR) for continuous variables and frequencies and proportions for categorical variables. A KruskalWallis test was performed for the comparison of plasma EFV concentrations among genotype groups. MannWhitney $U$-tests were used to compare plasma EFV concentrations between two genotypes. Wilcoxon matched pairs test was used for comparing two groups. A multivariable linear regression was performed for multiple factors including clinical characteristics, genotyping data with $p$ values less than 0.20. Statistical significance was defined as $p<0.05$ (STATA 14, StataCorp LP, TX) after non-adjusting and adjusting for $C Y P 2 B 6$ genotyping that is the main enzyme in efavirenz metabolism.

\section{Results}

\section{Demographic Characteristics, Allele and Genotype Frequencies}

A total of 149 participants were enrolled in the study. The average age of the patients was 37.4 (min-max; 19-59) years; $116(77.9 \%)$ patients were male and $33(22.1 \%)$ female. Patient demographics are presented in Table 1.

SULT1A1 copy number (CN) ranged from 1 to 6 copies and the frequency distribution of SULTIAI CN 1, 2, 3, 4, 5 , and 6 was $3.4 \%, 48.3 \%, 29.5 \%, 11.4 \%, 4.7 \%$, and $2.7 \%$, respectively. The observed SULTIAI gene deletion (1 copy) and multiallelic duplications were similar to previous reports in Thai, ${ }^{31}$ Caucasian-American, ${ }^{32}$ EuropeanCaucasian, ${ }^{33,34}$ Indians, ${ }^{35}$ Japanese, ${ }^{36}$ and Chinese. ${ }^{37}$

For UGT2B17, three distinct clusters representing zero (56.4\%), one (34.2\%) and two gene copies $(9.4 \%)$ were observed. Over $90 \%$ of patients were predicated to have decreased or no activity due to the loss of one or both gene copies. Furthermore, CNV frequencies differed from those reported by others. ${ }^{24,30,38-43}$ The distribution of SULT1A1 and $U G T 2 B 17$ gene copy number among different ethnic populations is shown in Table 2 .

Genotyping data for the investigated phase II drugmetabolizing enzyme and transporter genes were obtained for all 149 patients. Allele frequencies and copy number variations are summarized in Table 3 .

Table I Patient Demographics $(n=149)$

\begin{tabular}{|c|c|}
\hline $\begin{array}{l}\text { Data Characteristics, (Number of } \\
\text { Patients) at Baseline }\end{array}$ & Value \\
\hline HIV-I positive & $100 \%$ \\
\hline Age, mean $\pm S D$ (range; min-max), years & $37.4 \pm 8.54(19-59)$ \\
\hline $\begin{array}{l}\text { Gender, number (\%) } \\
\text {-Males } \\
\text {-Females }\end{array}$ & $\begin{array}{l}116(77.9 \%) \\
33(22.1 \%)\end{array}$ \\
\hline Body weight, mean $\pm \mathrm{SD}$ (min-max), $\mathrm{Kg}$ & $54.6 \pm 9.5(3 \mathrm{I}-86)$ \\
\hline CD4 cell count, median (IQR), cells/mm3 & $42(17-109)$ \\
\hline $\begin{array}{l}\text { Plasma HIV-I RNA, median (IQR), Log } \\
\text { copies/mL }\end{array}$ & $5.8(5.4-6.2)$ \\
\hline Hemoglobin, mean $\pm S D, g / d L$ & $10.8 \pm 1.8(6.5-15.5)$ \\
\hline Blood Urea Nitrogen, median (IQR), mg/dL & $10(7-11)$ \\
\hline Serum creatinine, mean $\pm \mathrm{SD}$ (min-max), $\mathrm{mg} / \mathrm{dL}$ & $0.7 \pm 0.2(0.3-1.3)$ \\
\hline Direct bilirubin, median (IQR), mg/dL & $0.2(0.1-0.4)$ \\
\hline Total bilirubin, median (IQR), mg/dL & $0.5(0.3-0.7)$ \\
\hline Alkaline phosphatase, median (IQR), mg/dL & $105(74-167)$ \\
\hline $\begin{array}{l}\text { Aspartate aminotransferase, median (IQR), } \\
\mathrm{U} / \mathrm{L}\end{array}$ & $37(27-52)$ \\
\hline Alanine aminotransferase, median (IQR), U/L & $31(19-46)$ \\
\hline
\end{tabular}


Table 2 SULTIAI and UGT2BI7 Gene Copy Number (CN) Distribution Among Different Ethnic Populations

\begin{tabular}{|c|c|c|c|c|c|c|c|c|c|}
\hline $\begin{array}{l}\text { SULTIAI gene } \\
\text { copy number }\end{array}$ & $\begin{array}{l}\text { Present } \\
\text { study } \\
(n=149)\end{array}$ & $\begin{array}{c}\text { Charoenchokthavee } \\
\text { et al } 2016^{31}\end{array}$ & $\begin{array}{c}\text { Hebbring } \\
\text { et al } \\
2007^{32}\end{array}$ & $\begin{array}{l}\text { Gjerde } \\
\text { et al } \\
2008^{33}\end{array}$ & $\begin{array}{c}\text { Gaedigk } \\
\text { et al } \\
2012^{30}\end{array}$ & $\begin{array}{c}\text { Tremmel } \\
\text { et al } \\
2017^{34}\end{array}$ & $\begin{array}{c}\text { Almal } \\
\text { et al } \\
2017^{35}\end{array}$ & $\begin{array}{l}\text { Yu et al } \\
2013^{36}\end{array}$ & $\begin{array}{l}\text { Li et al } \\
2010^{37}\end{array}$ \\
\hline Ethnicity & Thai & Thai & $\begin{array}{l}\text { Caucasian- } \\
\text { American }\end{array}$ & $\begin{array}{l}\text { European- } \\
\text { Caucasian }\end{array}$ & Caucasian & $\begin{array}{l}\text { European- } \\
\text { Caucasian }\end{array}$ & Indian & Japanese & Chinese \\
\hline I & $3.4 \%$ & $0 \%$ & $4.7 \%$ & $5.3 \%$ & $4.3 \%$ & $3.3 \%$ & $3.8 \%$ & $0 \%$ & $1.7 \%$ \\
\hline 2 & $48.3 \%$ & $97.1 \%$ & $69.6 \%$ & $64.9 \%$ & $47.2 \%$ & $60.3 \%$ & $64.9 \%$ & $65 \%$ & $75.7 \%$ \\
\hline 3 & $29.5 \%$ & $2.9 \%$ & $21 \%$ & $21.8 \%$ & $35.4 \%$ & $29.8 \%$ & $21.7 \%$ & $25.8 \%$ & NA \\
\hline 4 & $11.4 \%$ & $0 \%$ & $3.6 \%$ & $6 \%$ & $9.3 \%$ & NA & $5.1 \%$ & NA & NA \\
\hline 5 & $4.7 \%$ & $0 \%$ & NA & $2 \%$ & $3.5 \%$ & NA & NA & NA & NA \\
\hline 6 & $2.7 \%$ & $0 \%$ & NA & $0 \%$ & $0.3 \%$ & NA & NA & NA & NA \\
\hline$>2$ & $48.3 \%$ & $2.9 \%$ & $25.7 \%$ & $29.8 \%$ & $48.5 \%$ & $36.4 \%$ & $31.3 \%$ & $35 \%$ & $22.6 \%$ \\
\hline$\geq 4$ & $18.8 \%$ & NA & $4.7 \%$ & $8 \%$ & $13.1 \%$ & $6.6 \%$ & $\begin{array}{l}\mathrm{CN}>4 \\
(4.5 \%)\end{array}$ & $9.2 \%$ & $22.6 \%$ \\
\hline $\begin{array}{l}\text { UGT2BI7 } \\
\text { gene copy } \\
\text { number }\end{array}$ & & $\begin{array}{c}\text { Angstadt et al } \\
2013^{41}\end{array}$ & $\begin{array}{l}\text { Chew } \\
\text { et al } \\
2011^{42}\end{array}$ & $\begin{array}{c}\text { Haas et al } \\
2014^{24}\end{array}$ & $\begin{array}{c}\text { Gaedigk } \\
\text { et al } \\
2012^{30}\end{array}$ & $\begin{array}{c}\text { Uddin } \\
\text { et al } \\
2013^{43}\end{array}$ & $\begin{array}{c}\text { Song } \\
\text { et al } \\
2017^{66}\end{array}$ & $\begin{array}{l}\text { Mafune } \\
\text { et al } \\
2015^{40}\end{array}$ & $\begin{array}{c}\text { Yang } \\
\text { et al } \\
2008^{39}\end{array}$ \\
\hline Ethnicity & & American & Caucasian & $\begin{array}{c}\text { Hispanic\&Non- } \\
\text { Hispanic- } \\
\text { American }\end{array}$ & Caucasian & $\begin{array}{l}\text { European- } \\
\text { Caucasian }\end{array}$ & Chinese & Japanese & Chinese \\
\hline 0 & $56.4 \%$ & $14 \%$ & $12 \%$ & $6 \%$ & $11.6 \%$ & $13 \%$ & NA & $80 \%$ & $76.8 \%$ \\
\hline 1 & $34.2 \%$ & $47.8 \%$ & $45.5 \%$ & $43 \%$ & $46.7 \%$ & $51 \%$ & $\begin{array}{l}\mathrm{CN}<2 \\
(73 \%)\end{array}$ & $19 \%$ & $21.6 \%$ \\
\hline 2 & $9.4 \%$ & $38.2 \%$ & $42.5 \%$ & $49 \%$ & $40.6 \%$ & $35 \%$ & $27 \%$ & $1 \%$ & $1.6 \%$ \\
\hline$>2$ & $0 \%$ & $0 \%$ & $0 \%$ & $1 \%$ & $1.2 \%$ & $0 \%$ & $0 \%$ & $0 \%$ & $0 \%$ \\
\hline
\end{tabular}

Abbreviation: NA, not applicable.

All SNPs were in Hardy-Weinberg equilibrium $(p>0.05)$.

\section{Efavirenz Plasma Concentration in HIV-Infected Thai Adults}

The overall median steady state EFV plasma concentration (12 hours after dosing) was $2.41 \mathrm{mg} / \mathrm{L}$ (IQR; 1.46$4.12 \mathrm{mg} / \mathrm{L}$ ) at week 12 and $2.32 \mathrm{mg} / \mathrm{L}$ (IQR; 1.54$3.70 \mathrm{mg} / \mathrm{L})$ at week 24 . Of the 149 patients, $100(67.1 \%)$ were within the therapeutic range (1 to $4 \mathrm{mg} / \mathrm{L})$. Large inter-individual variation in EFV plasma concentrations was observed for 49 patients (32.9\%), ranging from $<1 \mathrm{mg} / \mathrm{L}$ (efficacy cut-off value) in 11 cases $(7.4 \%)$ to $>4 \mathrm{mg} / \mathrm{L}$ (toxicity cut-off value) in 38 cases $(25.5 \%)$ at week 12 , and 15 cases with $<1 \mathrm{mg} / \mathrm{L}$ and 34 cases with $>4 \mathrm{mg} / \mathrm{L}$ at week 24 .
Relationship Between SNPs Tested, Gene Copy Number and Efavirenz Plasma Concentration

The SNP defining SULT1A1*2 (c.638G>A, Arg213His) was associated with plasma EFV concentrations at week 24 ( $p=0.048$ ) but was not significant for week 12 (Table 3, Figure 1A and B). Patients heterozygous for SULT1A1*2 had significantly lower median plasma EFV concentrations at week $24(2.18 \mathrm{mg} / \mathrm{L}$, IQR: 1.44-2.60) compared to patients who did not carry this variant $(2.33 \mathrm{mg} / \mathrm{L}$, IQR: 1.57-4.21). However, there was no significant effect on plasma EFV concentrations at week 12. An association was also observed for SULT1A1 gene copy number. Patients with $\mathrm{CN}>2$ predicted to have higher SULT1A1 activity were significantly associated with decreased plasma EFV concentrations at week $12(p=0.046)$. 
Table 3 Relationship Between Genetic Variation in Drug-Metabolizing Enzyme and Transporter Genes and Plasma Efavirenz Concentrations $(\mathrm{N}=149)$

\begin{tabular}{|c|c|c|c|c|}
\hline Gene & $\begin{array}{c}N=149 \\
(\%)\end{array}$ & $\begin{array}{l}\text { EFV Plasma Concentration } \\
\text { (mg/L), Median (IQR), Week I2 }\end{array}$ & $\begin{array}{l}\text { EFV Plasma Concentration } \\
\text { (mg/L), Median (IQR), Week } 24\end{array}$ & $\boldsymbol{P}_{\text {value }}{ }^{t}$ \\
\hline \multicolumn{5}{|l|}{$A B C A /$ c. $4760 A>G(r s 2230808)$} \\
\hline $\mathrm{A} / \mathrm{A}$ & $32(21.5)$ & $2.07(1.29-3.46)$ & $2.62(|.3|-3.8 I)$ & 0.601 \\
\hline $\mathrm{A} / \mathrm{G}$ & $69(46.3)$ & $2.23(1.34-3.53)$ & $2.33(\mathrm{I} .60-4.2 \mathrm{I})$ & 0.644 \\
\hline $\mathrm{G} / \mathrm{G}$ & $48(32.2)$ & $2.94(1.78-4.59)$ & $2.22(1.54-3.10)$ & 0.056 \\
\hline$P$-value & & 0.103 & 0.775 & \\
\hline \multicolumn{5}{|l|}{$A B C C 2$ g.6823। $A>G(r s 3740065)$} \\
\hline $\mathrm{A} / \mathrm{A}$ & $63(42.3)$ & $2.43(1.57-4.10)$ & $2.22(I .54-3.4 I)$ & 0.059 \\
\hline $\mathrm{A} / \mathrm{G}$ & $86(57.7)$ & $2.40(I .36-4.2 I)$ & $2.38(1.55-4.01)$ & 0.728 \\
\hline$P$-value & & 0.476 & 0.596 & \\
\hline \multicolumn{5}{|l|}{$A B C C 2$ c. $-24 C>T(r s 717620)$} \\
\hline $\mathrm{C} / \mathrm{C}$ & $88(59.1)$ & $2.43(1.52-4.26)$ & $2.50(1.56-3.84)$ & 0.943 \\
\hline $\mathrm{C} / \mathrm{T}$ & $56(37.6)$ & $2.23(1.4 \mid-4.14)$ & $2.22(1.44-4.04)$ & 0.118 \\
\hline $\mathrm{T} / \mathrm{T}$ & $5(3.3)$ & $2.29(1.57-2.38)$ & $2.13(1.58-3.10)$ & 0.225 \\
\hline$P$-value & & 0.688 & 0.721 & \\
\hline \multicolumn{5}{|l|}{$A B C C 4$ c.3348A>G (rs|75I034) } \\
\hline $\mathrm{A} / \mathrm{A}$ & $97(65.1)$ & $2.41(1.57-4.99)$ & $2.36(1.55-3.67)$ & 0.526 \\
\hline $\mathrm{A} / \mathrm{G}$ & $45(30.2)$ & $2.29(1.43-5.02)$ & $2.33(1.56-4.24)$ & 0.343 \\
\hline $\mathrm{G} / \mathrm{G}$ & $7(4.7)$ & $2.43(1.03-2.73)$ & $1.81(1.07-2.46)$ & 0.735 \\
\hline$P$-value & & 0.516 & 0.322 & \\
\hline \multicolumn{5}{|l|}{ SULTIAI c.638G>A (rs|042028) } \\
\hline $\mathrm{G} / \mathrm{G}$ & $123(82.6)$ & $2.39(1.4 I-4.54)$ & $2.33(1.57-4.21)$ & 0.629 \\
\hline $\mathrm{G} / \mathrm{A}$ & $26(17.4)$ & $2.41(1.48-3.22)$ & $2.18(1.44-2.60)$ & 0.109 \\
\hline$P$-value & & 0.638 & $0.048^{\ddagger}$ & \\
\hline \multicolumn{5}{|l|}{ SULTIAI copy number (CN) } \\
\hline $\mathrm{I}$ & $5(3.4)$ & $2.43(1.34-4.97)$ & $2.46(2.22-2.55)$ & 0.500 \\
\hline 2 & $72(48.3)$ & $2.78(1.64-4.24)$ & $2.32(1.6 I-4.01)$ & 0.143 \\
\hline 3 & $44(29.5)$ & $2.06(1.26-2.82)$ & $2.22(1.47-4.02)$ & 0.524 \\
\hline 4 & $17(11.4)$ & $2.02(1.43-3.89)$ & $2.30(1.47-3.00)$ & 0.756 \\
\hline 5 & $7(4.7)$ & $2.77(1.33-5.84)$ & $2.72(1.09-5.58)$ & 0.398 \\
\hline 6 & $4(2.7)$ & $3.95(2.45-5.32)$ & $2.10(1.60-2.98)$ & 0.068 \\
\hline$>2$ & $72(48.3)$ & $2.07(1.39-3.22)$ & $2.30(\mid .52-3.5 I)$ & 0.860 \\
\hline$P$-value & & 0.194 & 0.958 & \\
\hline \multicolumn{5}{|l|}{ UGT2B7 c.-16IC>T (rs7668258) } \\
\hline $\mathrm{C} / \mathrm{C}$ & $88(59.1)$ & $2.20(1.45-3.94)$ & $2.22(1.57-3.50)$ & 0.126 \\
\hline $\mathrm{C} / \mathrm{T}$ & $52(34.9)$ & $2.78(1.56-4.78)$ & $2.33(1.52-4.24)$ & 0.536 \\
\hline $\mathrm{T} / \mathrm{T}$ & $9(6)$ & $2.15(1.37-2.55)$ & $2.85(1.47-3.87)$ & $0.012^{\ddagger}$ \\
\hline$P$-value & & 0.269 & 0.776 & \\
\hline \multicolumn{5}{|l|}{ UGT2B7 c.2IIG>T (rs|22337|9) } \\
\hline G/G & $124(83.2)$ & $2.43(1.52-4.12)$ & $2.30(1.54-3.59)$ & 0.137 \\
\hline $\mathrm{G} / \mathrm{T}$ & $24(16.1)$ & $\mathrm{I} .74(\mathrm{I} .36-3.4 \mathrm{I})$ & $2.46(1.95-4.19)$ & 0.317 \\
\hline $\mathrm{T} / \mathrm{T}$ & $\mathrm{I}(0.7)$ & $4.63(4.63-4.63)$ & $4.17(4.17-4.17)$ & 0.317 \\
\hline$P$-value & & 0.353 & 0.462 & \\
\hline
\end{tabular}


Table 3 (Continued).

\begin{tabular}{|c|c|c|c|c|}
\hline Gene & $\begin{array}{c}N=149 \\
(\%)\end{array}$ & $\begin{array}{l}\text { EFV Plasma Concentration } \\
\text { (mg/L), Median (IQR), Week I } 2\end{array}$ & $\begin{array}{l}\text { EFV Plasma Concentration } \\
\text { (mg/L), Median (IQR), Week } 24\end{array}$ & $\boldsymbol{P}$ value $^{t}$ \\
\hline \multicolumn{5}{|c|}{ UGT2B7 c.372A>G (rs28365063) } \\
\hline $\mathrm{A} / \mathrm{A}$ & $80(53.7)$ & $2.57(1.39-4.16)$ & $2.22(1.42-3.50)$ & 0.094 \\
\hline $\mathrm{A} / \mathrm{G}$ & $59(39.6)$ & $2.4 I(I .69-3.5 I)$ & $2.55(1.84-4.42)$ & 0.786 \\
\hline $\mathrm{G} / \mathrm{G}$ & $10(6.7)$ & $1.58(1.30-4.63)$ & $2.22(1.36-4.17)$ & 0.876 \\
\hline$P$-value & & 0.720 & $0.27 I$ & \\
\hline \multicolumn{5}{|c|}{ UGT2BI 7 copy number } \\
\hline 0 & $84(56.4)$ & $2.42(1.46-3.92)$ & $2.37(1.54-3.64)$ & 0.450 \\
\hline I & $51(34.2)$ & $2.38(1.48-4.10)$ & $2.22(1.52-3.59)$ & 0.297 \\
\hline 2 & $14(9.4)$ & $2.84(1.24-5.42)$ & $2.63(2.05-5.52)$ & 0.875 \\
\hline $0-1$ & $135(90.6)$ & $2.41(1.48-3.99)$ & $2.30(1.53-3.62)$ & 0.216 \\
\hline$P$-value & & 0.969 & 0.300 & \\
\hline
\end{tabular}

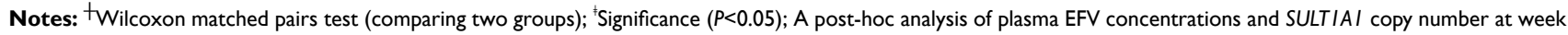
12 was as follows: $\mathrm{CN}=2$ vs $\mathrm{CN} \geq 3(P=0.046), \mathrm{CN}=2$ vs $\mathrm{CN}=3(p=0.019), \mathrm{CN}=2$ vs $\mathrm{CN}=3+4(P=0.015)$.

Table 4 Univariate and Multivariate Analyses of Genetic and Non-Genetic Factors Associated with Plasma Efavirenz Concentrations at Week 12 and 24 in HIV-I Infected Thai Adults

\begin{tabular}{|c|c|c|c|c|c|c|c|c|}
\hline \multirow[t]{3}{*}{ Characteristics/Duration } & \multicolumn{4}{|c|}{ Week 12} & \multicolumn{4}{|c|}{ Week 24} \\
\hline & \multicolumn{2}{|c|}{ Univariate Analysis } & \multicolumn{2}{|c|}{ Multivariate Analysis } & \multicolumn{2}{|c|}{ Univariate Analysis } & \multicolumn{2}{|c|}{ Multivariate Analysis } \\
\hline & Beta & $P$ value & Beta & $P$ value & Beta & $P$ value & Beta & $P$ value \\
\hline Age, years & 0.13 & 0.115 & & & 0.04 & 0.628 & & \\
\hline Gender & 0.04 & 0.653 & & & 0.11 & 0.181 & & \\
\hline Body weight, kg & -0.04 & 0.634 & & & 0.01 & 0.883 & & \\
\hline Height, cm & -0.16 & 0.048 & & & -0.04 & $0.68 I$ & & \\
\hline Hemoglobin, g/dL & -0.04 & 0.622 & & & -0.23 & 0.009 & -0.27 & 0.002 \\
\hline Blood Urea Nitrogen, mg/dL & -0.01 & 0.948 & & & 0.02 & 0.865 & & \\
\hline Serum creatinine, $\mathrm{mg} / \mathrm{dL}$ & -0.06 & 0.504 & & & -0.21 & 0.016 & -0.25 & 0.004 \\
\hline Albumin, $g / d L$ & -0.01 & 0.965 & & & -0.01 & 0.887 & & \\
\hline Globulin, g/dL & 0.19 & 0.022 & 0.20 & 0.018 & 0.19 & 0.030 & & \\
\hline Direct bilirubin, mg/dL & -0.05 & 0.555 & & & 0.03 & 0.721 & & \\
\hline Total bilirubin, mg/dL & -0.06 & 0.495 & & & 0.02 & 0.806 & & \\
\hline Alkaline phosphatase, U/L & 0.03 & 0.747 & & & 0.01 & 0.963 & & \\
\hline Aspartate aminotransferase, U/L & -0.06 & 0.488 & & & -0.09 & 0.339 & & \\
\hline Alanine aminotransferase, $\mathrm{U} / \mathrm{L}$ & -0.08 & 0.360 & & & -0.09 & 0.313 & & \\
\hline$A B C A I$ (c.4760A $>G$, rs2230808) & 0.02 & 0.791 & & & -0.06 & 0.458 & & \\
\hline$A B C C 2(\mathrm{~g} .6823 \mid \mathrm{A}>\mathrm{G}, \mathrm{rs} 3740065)$ & -0.01 & 0.905 & & & 0.07 & 0.423 & & \\
\hline$A B C C 2$ (c. $-24 C>T$, rs7। 7620$)$ & -0.03 & 0.687 & & & -0.07 & 0.437 & & \\
\hline$A B C C 4(c .3348 A>G, r s \mid 751034)$ & -0.01 & 0.988 & & & -0.07 & 0.398 & & \\
\hline SULTIAI*2 (c.638G>A, rsI042028) & -0.10 & 0.220 & & & -0.19 & 0.027 & -0.17 & 0.049 \\
\hline SULTIAI copy number & -0.11 & 0.204 & & & -0.05 & 0.595 & & \\
\hline UGT2B7 (c.-I6IC>T, rs7668258) & -0.01 & 0.994 & & & 0.11 & 0.207 & & \\
\hline UGT2B7, (c.2IIG>T, rs/22337|9) & -0.01 & 0.898 & & & 0.08 & 0.359 & & \\
\hline UGT2B7, (c.372A>G, rs28365063) & -0.04 & 0.601 & & & 0.07 & 0.421 & & \\
\hline UGT2BI 7 copy number & 0.07 & 0.395 & & & 0.02 & 0.774 & & \\
\hline
\end{tabular}

Abbreviations: CD4, cluster of differentiation 4; HIV, human immunodeficiency virus; RNA, ribonucleic acid; ARV, antiretroviral; HDL, high-density lipoprotein; LDL, lowdensity lipoprotein; ABCBI, ATP Binding Cassette Subfamily B Member I; ABCAI, ATP binding cassette subfamily A member I; ABCC2, ATP Binding Cassette Subfamily C Member 2; SULTIAI, sulphotransferase IAI; UGT2B7, UDP Glucuronosyltransferase Family 2 Member B7. 

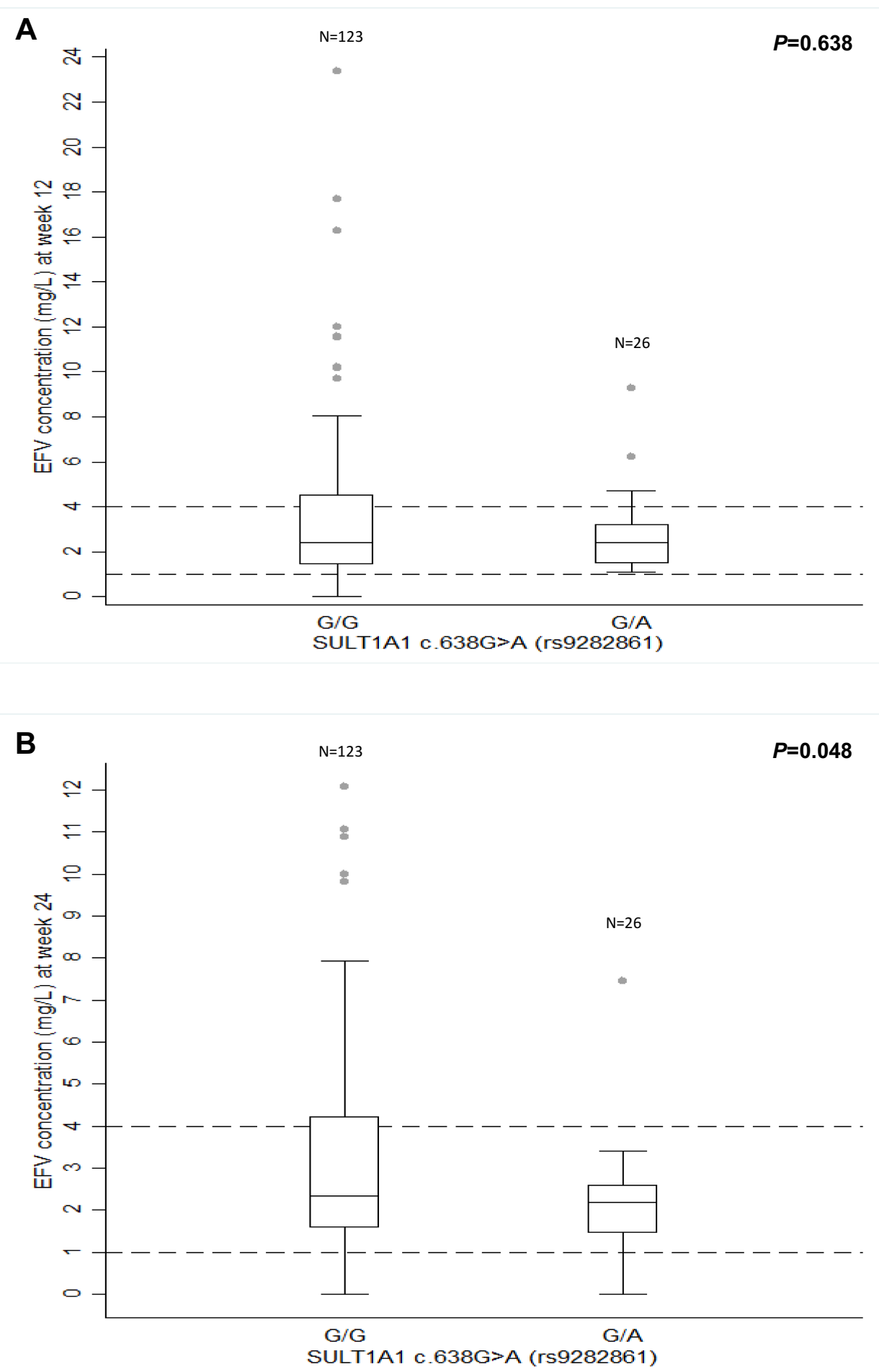

Figure I Influence of SULTIAI*2 (c.638G>A) on median plasma efavirenz (EFV) concentrations at week 12 and 24. Dash lines represent the therapeutic window for EFV (I$4 \mathrm{mg} / \mathrm{L}$ ). (A) Median plasma EFV concentrations compared between groups $(p=0.638)$ at week 12. (B) Median plasma EFV concentrations were significantly lower in heterozygous patients $(638 \mathrm{G} / \mathrm{A} ; 2.18 \mathrm{mg} / \mathrm{L}$, IQR I.44-2.60, p=0.048) compared to those not carrying the variant (638G/G; $2.33 \mathrm{mg} / \mathrm{L}$, IQR I.57-4.2I).

A comparison of SULT1A1 CN and plasma EFV concentrations at week 12 was as follows: $\mathrm{CN}=3$ versus $\mathrm{CN}=2$ ( $p=0.019), \mathrm{CN}=4$ versus $\mathrm{CN}=2(p=0.192)$, and $\mathrm{CN}=2$ versus $\mathrm{CN}=3$ and $4(p=0.015)$, respectively (Figure 2$)$.
Copy number of UGT2B17 was not associated with plasma EFV concentrations.

We also observed a trend for $A B C A 14760 \mathrm{G} / \mathrm{G}$ and higher median EFV concentrations $(2.94 \mathrm{mg} / \mathrm{L}$, IQR: 


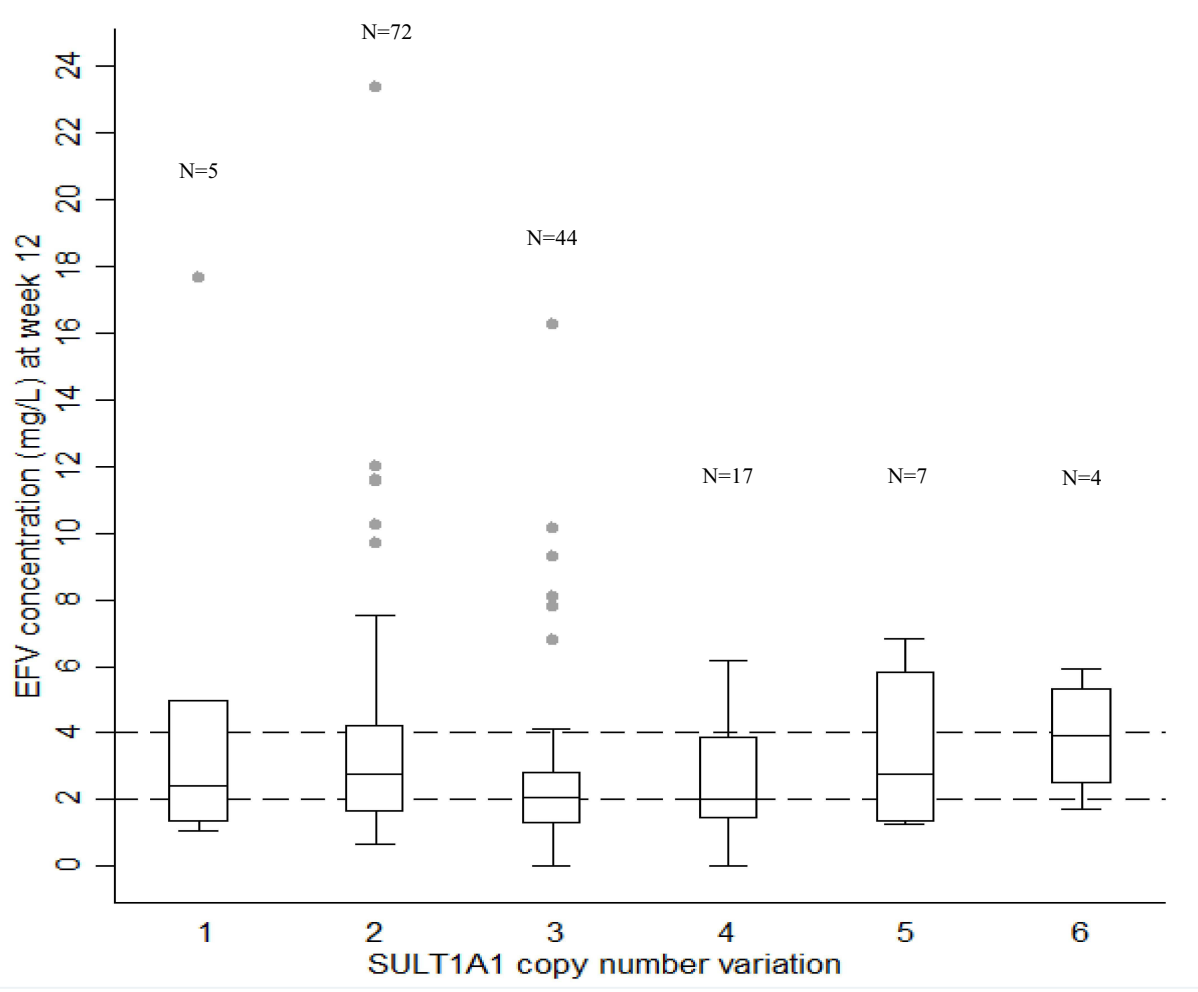

Figure 2 Influence of SULTIAI copy number variation on median plasma efavirenz (EFV) concentrations at week 12. Dash lines represent the therapeutic window for EFV (I-4 mg/L). A comparison of SULTIAI copy number $(\mathrm{CN})$ and median plasma EFV concentration at week I2. Median plasma EFV concentrations were significantly lower in patients with $\mathrm{CN} \geq 3(p=0.046), \mathrm{CN}=3(p=0.019)$, and $\mathrm{CN}=3+4(p=0.015)$ compared to those carrying $\mathrm{CN}=2$.

1.78-4.59) compared to subjects homozygous for the reference "A" allele $(2.07 \mathrm{mg} / \mathrm{L}, 1.29-3.46), p=0.059$. Conversely, patients genotyped as $A B C C 4 \quad 3348 \mathrm{G} / \mathrm{G}$ tended to have lower EFV concentrations $(1.81 \mathrm{mg} / \mathrm{L}$, IQR: 1.07-2.46) compared to those homozygous for the reference "A" (2.36 mg/L, IQR: 1.55-3.67), $p=0.140$.

In a univariate linear regression model, height, and globulin were significantly associated with plasma EFV concentrations at week 12 and hemoglobin, serum creatinine, globulin, and SULTIA1*2 were significantly associated with plasma EFV concentrations at week 24 $(p<0.05)$. Our previous studies reported that $C Y P 2 B 6$ genotypes were significantly associated with higher or lower plasma EFV concentrations, ${ }^{15,28,29}$ thus, this variable was used in the composition of the multiple regression analysis. In a multivariable analysis of non-adjusting for CYP2B6 genotype, globulin was significantly associated with plasma EFV concentrations at week $12(p=0.018)$ and hemoglobin, serum creatinine, and SULT1A1*2 allele were significantly associated with plasma EFV concentrations at week $24(p=0.049)$, as presented in Table 4 . After adjusting for $C Y P 2 B 6$ genotype that is a main factor for EFV metabolism in the multivariable linear regression analysis, a trend toward statistical significance of SULT1A1*2 was observed for patients carrying the SULT1A1*2 allele (c.638G $>A$ ), $p=0.089$. However, hemoglobin and serum creatinine were still significantly associated with plasma EFV concentrations at week 24 ( $p=0.006, p=0.010$, respectively). Potential differences in demographic and clinical characteristics were also compared between SULT1A1*2 subgroups $(* 1 / * 1, * 1 / * 2)$; however, statistically significant differences in these data between the subgroups were not found.

\section{Discussion}

Previous studies have established that CYP2B6 is a major route to EFV metabolism and thus plays an important role in EFV pharmacokinetics and pharmacodynamics. The Clinical Pharmacogenetics Implementation Consortium (CPIC) has recently published a CYP2B6-efavirenz guideline. ${ }^{44}$ However, despite the prominent role of CYP2B6, notable portions of variability in plasma EFV concentrations cannot be explained by genetic variation in CYP2B6. In this study, we set out to explore whether 
minor polymorphic phase II pathways (SULT1A1, $U G T 2 B 7$ and/or drug transporters $A B C A 1, A B C C 2$ and $A B C C 4$ ) may also contribute to the observed variability. ${ }^{17}$

To that end, eight SNPs were selected in genes that have been shown to influence EFV pharmacokinetics ${ }^{22,23,45,46}$ and, therefore, may also be involved in EFV transport and/or metabolism. Notably, SULT1A1 was previously implicated to be significantly associated with plasma EFV concentrations, ${ }^{24}$ although its effects on EFV PK remain unknown. The most striking result was the observation that the SULT1A1*2 (c.638G>A) allele and SULT1A1 copy number variation were associated with reduced plasma EFV concentrations. The nonsynonymous SNP causes an $\operatorname{Arg} 213$ His amino acid substitution and is found at $8.7 \%$ in this study, similar to that of other East Asian populations (8\%, $12 \%, 17 \%$ in Han Chinese, Korean, Japanese, respectively), while high allelic frequency is shown at $33 \%, 29 \%$ in Caucasian-American and African-Americans. ${ }^{47-49}$ The Arg213His substitution negatively affects enzyme activity by decreasing protein thermostability. ${ }^{50,51}$ The SULT1A1*2 allele has been associated with decreased estrogen metabolism and hormone-dependent cancers and has also been described to contribute to the risk of cancer or response to therapy. ${ }^{52-54}$ There is no direct evidence supporting that genetic variation of SULTIA1 contributes to EFV PK, but Haas et $\mathrm{al}^{24}$ have reported a significant relationship between SULT1A1 c.667A $>$ G (Met223Val, rs1801030) and plasma EFV concentrations. This study was, however, limited due to the lack of correction for multiple comparisons and another limitation of the Haas et $\mathrm{al}^{24}$ study is that SULT1A1 copy number variation was not accounted for pharmacokinetics of EFV. However, SULT1A1 CNVs have been shown to be the most important underlying genetic variation determining SULT1A1 activity ${ }^{55}$ and have been shown to impact the metabolism of several drugs. ${ }^{36,56,57}$ Our data suggest that SULT1A1 copy number and plasma EFV concentrations are indeed correlated contrasting the conclusion drawn by Hass et al. ${ }^{24}$ Patients carrying more than 2 SULT1A1 gene copies had decreased plasma EFV concentrations which were especially evident for subjects with 3 gene copies, the second most frequent CNV allele in our cohort. Based on our findings, the addition of SULTIA1 $\mathrm{CNV}$ testing is strongly encouraged to independently confirm the contribution of this pharmacogene to EFV metabolism and determine whether SULT1Al genotype is a clinically useful marker to predict outcome and/or reduce adverse effects. No correlations were detected for UGT2B17 copy number.
The SULT1A1 CNV frequencies were markedly different from those previously reported in Thai, ${ }^{31}$ a small study that only included 34 subjects. The frequencies we describe here are overall consistent with those found across other populations (Table 2). Regarding the frequency of UGT2B17 copy number, there appears to be considerable variation among the major population groups. ${ }^{24,30,39-43}$ The frequencies we report for our cohort are distinct from those described for other Asian populations, but share the overall lower frequencies of 2-copy alleles compared to populations of European descent.

We also observed trends of association between variants in $A B C A 1, A B C C 4$ transporter genes and EFV concentrations which were consistent with previous reports. ${ }^{11,22,58} A B C C 4$ c. $3348 \mathrm{~A}>\mathrm{G}$ carriers (MRP4, multidrug resistance protein 4) had a tendency towards lower plasma EFV concentrations. This transporter, also known as MRP4 is located in the blood-brain barrier and in the kidney. ${ }^{59}$ MRP4 is mainly expressed in the blood-facing membrane of the brain capillaries and choroid plexus facilitating the export of substrate drugs via luminal cells. ${ }^{60}$ The c.3348A $>$ G SNP may reduce expression concentrations and thereby increase EFV concentrations in cerebrospinal fluid which may in turn cause central nervous system effects. ${ }^{61}$ Many SNPs of $A B C C 4$ have previously been reported to correlate with EFV PK parameters. ${ }^{22}$ However, it remains unclear whether genetic variation of $A B C C 4$ has clinical relevance.

Given the complexity of HIV-1 infection, it is not surprising that multiple factors including patient demographics such as gender, age, body weight, liver, and renal impairment influence plasma EFV concentration. ${ }^{62}$ The contribution of these factors is in line with our previous findings ${ }^{63}$ as well as the current study (ie, associations between plasma EFV concentrations and body weight, height, viral load, blood urea nitrogen (BUN) and aspartate aminotransferase (AST) were found). In addition, globulin influenced EFV metabolism at week 12 . At week 24 , however, there were only three factors, hemoglobin, serum creatinine, and SULTIA1*2 that remained statistically significant with low plasma EFV concentration after multivariate analysis. Lastly, EFV therapy may also influence hemoglobin and albumin, ${ }^{64}$ and Yimer et $\mathrm{al}^{65}$ reported that lower baseline albumin levels are an important predictor for EFV-based HAART-induced liver injury. Although power analysis of the effect size of SULT1A1*2 allele accounted for $17 \%$ of the observed variability in plasma EFV concentrations for multiple linear regression analysis by using non-adjusting for $C Y P 2 B 6$ genotyping model, this effect was disappeared after adjusting for $C Y P 2 B 6$ genotype in this 
study. The influence of cofactors of EFV biotransformation pathway may be reduced because the number of sample sizes in each group tends to be small. Therefore, the findings of this study need to be viewed as preliminary.

\section{Conclusion}

Our findings suggest that $S U L T 1 A 1 * 2$ and copy number variation contribute to the metabolism of drug regimens containing EFV. Thus, in addition to patient demographics and $C Y P 2 B 6$ genotype status, future investigations should include SULTIA1 to further our understanding of variability of EFV metabolism to ultimately empower us to more accurately predict EFV drug response and avoid adverse events.

\section{Acknowledgments}

This research is supported by the Faculty of Medicine, Ramathibodi Hospital, Mahidol University; The International Research Network-The Thailand Research Fund (IRN60W003), Thailand; Rachadapisek Sompote Fund for Postdoctoral Fellowship, Chulalongkorn University. The authors also thank the Division of Clinical Pharmacology, Toxicology \& Therapeutic Innovation, Children's Mercy Kansas City, and School of Medicine, University of Missouri-Kansas City, for providing access to quantitative multiplex PCR amplification.

\section{Disclosure}

The authors reported no conflicts of interest in this work.

\section{References}

1. Barusrux S, Urwijitaroon Y, Puapairoj C, Romphruk A, Sriwanitchrak P. Association of HCV and Treponema pallidum infection in HIV infected northeastern Thai male blood donors. $J$ Med Assoc Thai. 1997;80(Suppl 1):S106-111.

2. Mocroft A, Ledergerber B, Katlama C, et al. Decline in the AIDS and death rates in the EuroSIDA study: an observational study. Lancet. 2003;362(9377):22-29. doi:10.1016/S0140-6736(03)13802-0

3. Clevenbergh P, Mouly S, Sellier P, et al. Improving HIV infection management using antiretroviral plasma drug levels monitoring: a clinician's point of view. Curr HIV Res. 2004;2(4):309-321. doi:10.2174/1570162043351129

4. Fabbiani M, Bracciale L, Ragazzoni E, et al. Relationship between antiretroviral plasma concentration and emergence of HIV-1 resistance mutations at treatment failure. Infection. 2011;39(6):563-569. doi:10.1007/s15010-011-0183-8

5. Langmann P, Weissbrich B, Desch S, et al. Efavirenz plasma levels for the prediction of treatment failure in heavily pretreated HIV-1 infected patients. Eur J Med Res. 2002;7(7):309-314.

6. Marzolini C, Telenti A, Decosterd LA, Greub G, Biollaz J, Buclin T. Efavirenz plasma levels can predict treatment failure and central nervous system side effects in HIV-1-infected patients. Aids. 2001;15 (1):71-75. doi:10.1097/00002030-200101050-00011
7. Pretorius E, Klinker H, Rosenkranz B. The role of therapeutic drug monitoring in the management of patients with human immunodeficiency virus infection. Ther Drug Monit. 2011;33(3):265-274. doi:10.1097/FTD.0b013e31821b42d1

8. Stahle L, Moberg L, Svensson JO, Sonnerborg A. Efavirenz plasma concentrations in HIV-infected patients: inter- and intraindividual variability and clinical effects. Ther Drug Monit. 2004;26 (3):267-270. doi:10.1097/00007691-200406000-00008

9. Arab-Alameddine M, Di Iulio J, Buclin T, et al. Pharmacogeneticsbased population pharmacokinetic analysis of efavirenz in HIV-1-infected individuals. Clin Pharmacol Ther. 2009;85 (5):485-494. doi:10.1038/clpt.2008.271

10. Cabrera SE, Santos D, Valverde MP, et al. Influence of the cytochrome P450 2B6 genotype on population pharmacokinetics of efavirenz in human immunodeficiency virus patients. Antimicrob Agents Chemother. 2009;53(7):2791-2798. doi:10.1128/AAC.01537-08

11. Csajka C, Marzolini C, Fattinger K, et al. Population pharmacokinetics and effects of efavirenz in patients with human immunodeficiency virus infection. Clin Pharmacol Ther. 2003;73(1):20-30. doi:10.1067/mcp.2003.22

12. Gounden V, van Niekerk C, Snyman T, George JA. Presence of the CYP2B6 516G $>$ T polymorphism, increased plasma Efavirenz concentrations and early neuropsychiatric side effects in South African HIVinfected patients. AIDS Res Ther. 2010;7:32. doi:10.1186/1742-6405-7-32

13. Sanchez A, Cabrera S, Santos D, et al. Population pharmacokinetic/ pharmacogenetic model for optimization of efavirenz therapy in Caucasian HIV-infected patients. Antimicrob Agents Chemother. 2011;55(11):5314-5324. doi:10.1128/AAC.00194-11

14. Desta Z, Saussele T, Ward B, et al. Impact of CYP2B6 polymorphism on hepatic efavirenz metabolism in vitro. Pharmacogenomics. 2007;8 (6):547-558. doi:10.2217/14622416.8.6.547

15. Sukasem C, Chamnanphon M, Koomdee N, et al. High plasma efavirenz concentration and CYP2B6 polymorphisms in Thai HIV-1 infections. Drug Metab Pharmacokinet. 2013;28(5):391-397. doi:10.2133/dmpk.DMPK-12-RG-120

16. Meng X, Yin K, Wang J, et al. Effect of CYP2B6 gene polymorphisms on efavirenz plasma concentrations in Chinese patients with HIV infection. PLoS One. 2015;10(6):e0130583. doi:10.1371/journal.pone. 0130583

17. Ward BA, Gorski JC, Jones DR, Hall SD, Flockhart DA, Desta Z. The cytochrome P450 2B6 (CYP2B6) is the main catalyst of efavirenz primary and secondary metabolism: implication for HIV/AIDS therapy and utility of efavirenz as a substrate marker of CYP2B6 catalytic activity. J Pharmacol Exp Ther. 2003;306(1):287-300. doi:10.1124/jpet.103.049601

18. Ogburn ET, Jones DR, Masters AR, Xu C, Guo Y, Desta Z. Efavirenz primary and secondary metabolism in vitro and in vivo: identification of novel metabolic pathways and cytochrome P450 2A6 as the principal catalyst of efavirenz 7-hydroxylation. Drug Metab Dispos. 2010;38(7):1218-1229. doi:10.1124/dmd.109.031393

19. Mutlib AE, Chen H, Nemeth GA, et al. Identification and characterization of efavirenz metabolites by liquid chromatography/ mass spectrometry and high field NMR: species differences in the metabolism of efavirenz. Drug Metab Dispos. 1999;27 (11):1319-1333.

20. Bae SK, Jeong YJ, Lee C, Liu KH. Identification of human UGT isoforms responsible for glucuronidation of efavirenz and its three hydroxy metabolites. Xenobiotica. 2011;41(6):437-444. doi:10.3109/ 00498254.2011.551849

21. Belanger AS, Caron P, Harvey M, Zimmerman PA, Mehlotra RK, Guillemette C. Glucuronidation of the antiretroviral drug efavirenz by UGT2B7 and an in vitro investigation of drug-drug interaction with zidovudine. Drug Metab Dispos. 2009;37(9):1793-1796. doi:10.1124/dmd.109.027706 
22. Sanchez-Martin A, Cabrera Figueroa S, Cruz R, et al. Gene-gene interactions between DRD3, MRP4 and CYP2B6 polymorphisms and its influence on the pharmacokinetic parameters of efavirenz in HIV infected patients. Drug Metab Pharmacokinet. 2016;31(5):349-355. doi:10.1016/j.dmpk.2016.06.001

23. Abla N, Chinn LW, Nakamura T, et al. The human multidrug resistance protein 4 (MRP4, ABCC4): functional analysis of a highly polymorphic gene. J Pharmacol Exp Ther. 2008;325(3):859-868. doi:10.1124/jpet.108.136523

24. Haas DW, Kwara A, Richardson DM, et al. Secondary metabolism pathway polymorphisms and plasma efavirenz concentrations in $\mathrm{HIV}$-infected adults with CYP2B6 slow metabolizer genotypes. J Antimicrob Chemother. 2014;69(8):2175-2182. doi:10.1093/jac/dku110

25. Sarfo FS, Zhang Y, Egan D, et al. Pharmacogenetic associations with plasma efavirenz concentrations and clinical correlates in a retrospective cohort of Ghanaian HIV-infected patients. J Antimicrob Chemother. 2014;69(2):491-499. doi:10.1093/jac/dkt372

26. Elens L, Vandercam B, Yombi JC, Lison D, Wallemacq P, Haufroid V. Influence of host genetic factors on efavirenz plasma and intracellular pharmacokinetics in HIV-1-infected patients. Pharmacogenomics. 2010;11(9):1223-1234. doi:10.2217/pgs.10.94

27. Cortes CP, Siccardi M, Chaikan A, Owen A, Zhang G, la Porte CJ. Correlates of efavirenz exposure in Chilean patients affected with human immunodeficiency virus reveals a novel association with a polymorphism in the constitutive androstane receptor. Ther Drug Monit. 2013;35(1):78-83. doi:10.1097/FTD.0b013e318274197e

28. Sukasem C, Cressey TR, Prapaithong P, et al. Pharmacogenetic markers of CYP2B6 associated with efavirenz plasma concentrations in HIV-1 infected Thai adults. Br J Clin Pharmacol. 2012;74 (6):1005-1012. doi:10.1111/j.1365-2125.2012.04288.x

29. Sukasem C, Manosuthi W, Koomdee N, et al. Low level of efavirenz in HIV-1-infected Thai adults is associated with the CYP2B6 polymorphism. Infection. 2014;42(3):469-474. doi:10.1007/s15010013-0560-6

30. Gaedigk A, Twist GP, Leeder JS. CYP2D6, SULT1A1 and UGT2B17 copy number variation: quantitative detection by multiplex PCR. Pharmacogenomics. 2012;13(1):91-111. doi:10.2217/pgs.11.135

31. Charoenchokthavee W, Ayudhya DP, Sriuranpong V, Areepium N. Effects of SULT1A1 copy number variation on estrogen concentration and tamoxifen-associated adverse drug reactions in premenopausal Thai breast cancer patients: a preliminary study. Asian Pac J Cancer Prev. 2016;17(4):1851-1855. doi:10.7314/APJCP.2016.17.4.1851

32. Hebbring SJ, Adjei AA, Baer JL, et al. Human SULT1A1 gene: copy number differences and functional implications. Hum Mol Genet. 2007;16(5):463-470. doi:10.1093/hmg/ddl468

33. Gjerde J, Hauglid M, Breilid H, et al. Effects of CYP2D6 and SULT1A1 genotypes including SULT1A1 gene copy number on tamoxifen metabolism. Ann Oncol. 2008;19(1):56-61. doi:10.1093/ annonc/mdm 434

34. Tremmel R, Herrmann K, Engst W, et al. Methyleugenol DNA adducts in human liver are associated with SULT1A1 copy number variations and expression levels. Arch Toxicol. 2017;91 (10):3329-3339. doi:10.1007/s00204-017-1955-4

35. Almal S, Padh H. SULT1A1 copy number variation: ethnic distribution analysis in an Indian population. Ann Hum Biol. 2017;44 (7):663-666. doi:10.1080/03014460.2017.1376852

36. Yu X, Kubota T, Dhakal I, et al. Copy number variation in sulfotransferase isoform 1A1 (SULT1A1) is significantly associated with enzymatic activity in Japanese subjects. Pharmgenomics Pers Med. 2013;6:19-24. doi:10.2147/PGPM.S36579

37. Li G, Ye C, Shu X-O, et al. Abstract 2857: SULT1A1 gene copy number variations and functional polymorphism in relation to breast cancer risk. Cancer Res. 2010;70(8 Supplement):2857. doi:10.1158/ 0008-5472.CAN-09-3903
38. Suktitipat B, Naktang C, Mhuantong W, et al. Copy number variation in Thai population. PLoS One. 2014;9(8):e104355-e104355. doi:10.1371/journal.pone.0104355

39. Yang T-L, Chen X-D, Guo Y, et al. Genome-wide copy-numbervariation study identified a susceptibility gene, UGT2B17, for osteoporosis. Am J Hum Genet. 2008;83(6):663-674. doi:10.1016/j. ajhg.2008.10.006

40. Mafune A, Hama T, Suda T, et al. Homozygous deletions of UGT2B17 modifies effects of smoking on TP53-mutations and relapse of head and neck carcinoma. BMC Cancer. 2015;15(1):205. doi:10.1186/s12885-015-1220-2

41. Angstadt AY, Berg A, Zhu J, et al. The effect of copy number variation in the phase II detoxification genes UGT2B17 and UGT2B28 on colorectal cancer risk. Cancer. 2013;119 (13):2477-2485. doi:10.1002/cncr.28009

42. Chew S, Mullin BH, Lewis JR, Spector TD, Prince RL, Wilson SG. Homozygous deletion of the UGT2B17 gene is not associated with osteoporosis risk in elderly Caucasian women. Osteoporos Int. 2011;22(6):1981-1986. doi:10.1007/s00198-010-1405-0

43. Uddin M, Maksymowych WP, Inman R, et al. UGT2B17 copy number gain in a large ankylosing spondylitis multiplex family. BMC Genet. 2013;14:67. doi:10.1186/1471-2156-14-67

44. Desta Z, Gammal RS, Gong L, et al. Clinical Pharmacogenetics Implementation Consortium (CPIC) guideline for CYP2B6 and efavirenz-containing antiretroviral therapy. Clin Pharmacol Ther. 2019;106(4):726-733. doi:10.1002/cpt.1477

45. Yu X, Dhakal IB, Beggs M, et al. Functional genetic variants in the 3'-untranslated region of sulfotransferase isoform 1A1 (SULT1A1) and their effect on enzymatic activity. Toxicol Sci. 2010;118 (2):391-403. doi:10.1093/toxsci/kfq296

46. Jakobsson J, Ekstrom L, Inotsume $\mathrm{N}$, et al. Large differences in testosterone excretion in Korean and Swedish men are strongly associated with a UDP-glucuronosyl transferase 2B17 polymorphism. J Clin Endocrinol Metab. 2006;91(2):687-693. doi:10.1210/jc.2005-1643

47. Carlini EJ, Raftogianis RB, Wood TC, et al. Sulfation pharmacogenetics: SULT1A1 and SULT1A2 allele frequencies in Caucasian, Chinese and African-American subjects. Pharmacogenetics. 2001;11(1):57-68. doi:10.1097/00008571200102000-00007

48. Lee SJ, Kim WY, Jarrar YB, Kim YW, Lee SS, Shin JG. Single nucleotide polymorphisms in SULT1A1 and SULT1A2 in a Korean population. Drug Metab Pharmacokinet. 2013;28(4):372-377. doi:10.2133/dmpk.DMPK-12-SC-110

49. Ohtake E, Kakihara F, Matsumoto N, et al. Frequency distribution of phenol sulfotransferase 1A1 activity in platelet cells from healthy Japanese subjects. Eur J Pharm Sci. 2006;28(4):272-277. doi:10.1016/j.ejps.2006.02.008

50. Raftogianis RB, Wood TC, Otterness DM, Van Loon JA, Weinshilboum RM. Phenol sulfotransferase pharmacogenetics in humans: association of common SULT1A1 alleles with TS PST phenotype. Biochem Biophys Res Commun. 1997;239(1):298-304. doi:10.1006/bbrc.1997.7466

51. Raftogianis RB, Wood TC, Weinshilboum RM. Human phenol sulfotransferases SULT1A2 and SULT1A1: genetic polymorphisms, allozyme properties, and human liver genotype-phenotype correlations. Biochem Pharmacol. 1999;58(4):605-616. doi:10.1016/S00062952(99)00145-8

52. Saintot M, Malaveille C, Hautefeuille A, Gerber M. Interactions between genetic polymorphism of cytochrome P450-1B1, sulfotransferase 1A1, catechol-o-methyltransferase and tobacco exposure in breast cancer risk. Int J Cancer. 2003;107(4):652-657. doi:10.1002/ijc.11432

53. Sillanpaa P, Kataja V, Eskelinen M, et al. Sulfotransferase 1A1 genotype as a potential modifier of breast cancer risk among premenopausal women. Pharmacogenet Genomics. 2005;15(10):749-752. doi:10.1097/01.fpc.0000172240.34923.46 
54. Shatalova EG, Walther SE, Favorova OO, Rebbeck TR, Blanchard RL. Genetic polymorphisms in human SULT1A1 and UGT1A1 genes associate with breast tumor characteristics: a case-series study. Breast Cancer Res. 2005;7(6):R909-R921. doi:10.1186/bcr1318

55. Hebbring SJ, Adjei AA, Baer JL, et al. Human SULT1A1 gene: copy number differences and functional implications. Hum Mol Genet. 2006;16(5):463-470.

56. Edavana VK, Yu X, Dhakal IB, et al. Sulfation of fulvestrant by human liver cytosols and recombinant SULT1A1 and SULT1E1. Pharmgenomics Pers Med. 2011;4:137-145. doi:10.2147/PGPM. S25418

57. Edavana VK, Dhakal IB, Yu X, Williams S, Kadlubar S. Sulfation of 4-hydroxy toremifene: individual variability, isoform specificity, and contribution to toremifene pharmacogenomics. Drug Metab Dispos. 2012;40(6):1210-1215. doi:10.1124/dmd.111.044040

58. Mukonzo JK, Owen JS, Ogwal-Okeng J, et al. Pharmacogeneticbased efavirenz dose modification: suggestions for an African population and the different CYP2B6 genotypes. PLoS One. 2014;9(1): e86919. doi:10.1371/journal.pone.0086919

59. Borst $\mathrm{P}$, de Wolf $\mathrm{C}$, van de Wetering $\mathrm{K}$. Multidrug resistance-associated proteins 3, 4, and 5. Pflugers Arch. 2007;453 (5):661-673. doi:10.1007/s00424-006-0054-9

60. Leggas M, Adachi M, Scheffer GL, et al. Mrp4 confers resistance to topotecan and protects the brain from chemotherapy. Mol Cell Biol. 2004;24(17):7612-7621. doi:10.1128/MCB.24.17.7612-7621.2004
61. Sanchez Martin A, Cabrera Figueroa S, Cruz Guerrero R, Hurtado LP, Hurle AD, Carracedo Alvarez A. Impact of pharmacogenetics on CNS side effects related to efavirenz. Pharmacogenomics. 2013;14(10):1167-1178. doi:10.2217/pgs.13. 111

62. Sukasem C, Churdboonchart V, Chasombat S, et al. Surveillance of genotypic resistance mutations in chronic HIV-1 treated individuals after completion of the National Access to Antiretroviral Program in Thailand. Infection. 2007;35(2):81-88. doi:10.1007/s15010-0076169-x

63. Sukasem C, Chamnanphon M, Koomdee N, et al. Pharmacogenetics and clinical biomarkers for subtherapeutic plasma efavirenz concentration in HIV-1 infected Thai adults. Drug Metab Pharmacokinet. 2014;29(4):289-295. doi:10.2133/dmpk.DMPK-13-RG-077

64. Metzger IF, Quigg TC, Epstein N, et al. Substantial effect of efavirenz monotherapy on bilirubin levels in healthy volunteers. Curr Ther Res Clin Exp. 2014;76:64-69. doi:10.1016/j.curtheres.2014.05.002

65. Yimer G, Amogne W, Habtewold A, et al. High plasma efavirenz level and CYP2B6*6 are associated with efavirenz-based HAARTinduced liver injury in the treatment of naive HIV patients from Ethiopia: a prospective cohort study. Pharmacogenomics J. 2012;12 (6):499-506. doi:10.1038/tpj.2011.34

66. Song R-H, Shao X-Q, Li L, Wang W, Zhang J-A. Copy number variations exploration of multiple genes in Graves' disease. Medicine. 2017;96(4):e5866. doi:10.1097/MD.0000000000005866

\section{Publish your work in this journal}

Pharmacogenomics and Personalized Medicine is an international, peer-reviewed, open access journal characterizing the influence of genotype on pharmacology leading to the development of personalized treatment programs and individualized drug selection for improved safety, efficacy and sustainability. This journal is indexed on the American Chemical Society's Chemical Abstracts Service (CAS). The manuscript management system is completely online and includes a very quick and fair peer-review system, which is all easy to use. Visit http://www.dovepress.com/testimonials.php to read real quotes from published authors. 